\title{
Desarrollo de la autonomía y la autorregulación en estudiantes universitarios: una experiencia de investigación y mediación
}

\section{The development of autonomy and self-regulation in university students: A research and mediation experience}

\section{IVET GARCÍA Montero* \\ Ruth Belinda Bustos CóRdova*}

El artículo presenta los resultados de una investigación realizada con estudiantes de la licenciatura en Intervención Educativa de la Universidad Pedagógica Nacional en Morelos, cuyo objetivo fue formar competencias para la autorregulación del aprendizaje en los aprendices y fortalecer sus procesos de autonomía, así como generar y sustentar teóricamente un sistema de acciones psicopedagógicas que medien el desarrollo de la autorregulación en el contexto de su formación profesional. El estudio se sustenta en el enfoque histórico cultural y en otras concepciones teóricas que explican la autorregulación del aprendizaje y la formación de aprendices autónomos, así como en el enfoque de investigación cualitativa; su diseño metodológico articula los principios del método genético y de la investigación-acción participativa. Los resultados avalan la relevancia de los procesos educativos y de las acciones de mediación psicopedagógica en el desarrollo de la autonomía y la autorregulación.

The article presents the results of research conducted with students of the career of Educational Intervention in the National Pedagogical University in Morelos. Its objective was to form competences for the self-regulation of apprenticeship learning and strengthen the students' autonomy in the learning processes, as well as theoretically generating and sustaining a system of psycho-pedagogical actions that mediate the development of self-regulation in the context of their professional training. The study is based on the historical-cultural approach and other theoretical conceptions that explain the self-regulation of learning and the formation of autonomous learners. It was carried out through the qualitative research approach and its methodological design articulates the principles of the genetic method and participatory action research. The results guarantee the relevance of educational processes and psychopedagogical mediation actions in the development of autonomy and self-regulation.

\section{Palabras clave:}

autorregulación del aprendizaje, competencias de autorregulación, autonomía, formación

\section{Keywords:}

self-regulation

of learning, self-regulation skills, autonomy, training

Recibido: 17 de febrero de 2020 | Aceptado para su publicación: 30 de junio de 2020| Fecha de publicación: 3 de julio de 2020

Recuperado de: https://sinectica.iteso.mx/index.php/SINECTICA/article/view/1108 doi: $10.31391 /$ S2007-7033(2020)0055-003

\footnotetext{
* Doctora en Enseñanza Superior. Profesora e investigadora de la Universidad Pedagógica Nacional (UPN). Representante del cuerpo académico Procesos de aprendizaje y formación docente en la diversidad de la UPN, Morelos. Perfil deseable de Prodep. Líneas de investigación: procesos de aprendizaje, autorregulación, educación superior y formación docente. Correo electrónico: ivet2010@gmail.com / https://orcid.org/0000-0003-4051-0278

** Doctora en Educación. Profesora e investigadora de la UPN. Integrante del cuerpo académico Procesos de aprendizaje y formación docente en la diversidad de la UPN, Morelos. Miembro del Sistema Nacional de Investigadores. Líneas de investigación: formación sociomoral, valores y diversidad, formación docente, educación indígena e intercultural. Correo electrónico: ruthbustos7@gmail.com / https://orcid.org/0000-0002-2047-177X
} 


\section{INTRODUCCIÓN}

$\mathrm{L}$

as condiciones actuales de existencia humana, permeadas por la vorágine de las tecnologías, la incertidumbre en las distintas esferas de inserción social y las exigencias socioeconómicas de carácter global imponen nuevas percepciones acerca de la enseñanza, el aprendizaje y las formas de lograrlo. Las personas requieren en la actualidad otras maneras de aprender que permitan la apropiación de los saberes necesarios para enfrentar tales exigencias; se habla así de una nueva "cultura del aprendizaje" (Pozo, 2008) caracterizada por demandar conocimientos múltiples y el aprendizaje permanente.

En este nuevo contexto de aprendizaje humano debe formarse a los educandos sobre la base del desarrollo de capacidades de aprendizaje y la apropiación de instrumentos psicológicos para su desempeño de manera autónoma. Las investigaciones recientes en el ámbito de la psicología educativa (Torrano, Fuentes y Soria, 2017) corroboran la importancia de las experiencias de aprendizaje que empoderan al aprendiz al posibilitar la formación de competencias para la autorregulación y la autonomía en el ámbito educativo.

En consecuencia, se hace imprescindible la profundización y reelaboración teórica que ayude a explicar los mecanismos de desarrollo de la autorregulación y la autonomía en el contexto educativo y, en la misma medida, analizar los problemas que impiden su formación. De igual modo, es la investigación psicoeducativa la vía para generar alternativas metodológicas aplicables en los procesos de enseñanza y de formación de los estudiantes que contribuyan al aprendizaje mismo de la autorregulación, al considerar que este es un proceso educable y que se pueden organizar acciones psicopedagógicas para favorecerlo (Schunk, 2012).

Los objetivos del estudio que este documento refiere respondieron a esa intención investigativa y estuvieron dirigidos a generar y sustentar teóricamente un sistema de acciones psicopedagógicas que medien el desarrollo de la autorregulación y la autonomía en el contexto de formación profesional de jóvenes que se forman para ser educadores. La investigación se planteó una interrogante esencial: ¿qué instrumentos psicológicos y acciones mediadoras de carácter psicopedagógico posibilitan el desarrollo de la autonomía y la apropiación de las competencias para la autorregulación del aprendizaje en el contexto de práctica real de los estudiantes que se forman en la Universidad Pedagógica Nacional (UPN)?

Como resultados fundamentales, el estudio permitió la identificación y el análisis de las problemáticas que inciden en los procesos de autonomía y autorregulación del aprendizaje, la formulación de un conjunto de propuestas de desarrollo por parte de los actores de la investigación, y la integración de un sistema de acciones psicopedagógicas mediadoras para la formación de competencias de autorregulación del aprendizaje y la autonomía que, una vez apropiadas por los aprendices, constituyen los instrumentos psicológicos internos que favorecen la autotransformación y el desarrollo de los propios estudiantes. 


\section{LOS PROCESOS DE AUTONOMÍA Y AUTORREGULACIÓN DEL APRENDIZAJE EN LA FORMACIÓN DE EDUCADORES}

A pesar de la reconocida necesidad de fortalecer la formación de los educadores y de desarrollar sus competencias para la autorregulación y la autonomía profesional, se registran escasas investigaciones en este ámbito, tanto a nivel internacional (Rosário et al., 2013) como nacional (Aguilar, 2020), cuestión que abre un camino necesario de búsqueda de alternativas psicopedagógicas para enriquecer esos procesos.

Las investigaciones revisadas para este estudio (Guzmán, Peña y Ramírez, 2015; Vargas, 2015) destacan la necesidad de transformar los mecanismos de preparación de los educadores y de introducir acciones mediadoras que contribuyan al desarrollo de la capacidad de autonomía, la reflexión crítica y la autorregulación de los docentes a lo largo de sus trayectorias formativas y, en consecuencia, replantear las acciones de los formadores de formadores en el marco de las instituciones que preparan al docente tanto a nivel inicial como en su desarrollo profesional continuo.

\section{MÉTODO DE INVESTIGACIóN}

El estudio está respaldado en los principios de la investigación cualitativa, por sus potencialidades en la comprensión de los procesos psicopedagógicos complejos (Bisquerra, 2014) y las posibilidades que brinda para estudiar estos fenómenos desde la perspectiva de los actores y del sentido que otorgan a sus propias experiencias, en este caso formativas.

En esta última dirección se fundamenta no solo la afiliación a la investigación cualitativa, sino la elección, en particular, del diseño de investigación-acción participativa (IAP), que garantizó un trayecto de sucesivos momentos de diagnóstico participativo, problematización y generación de alternativas encaminadas a la mejora y transformación de los procesos de aprendizaje autónomo y de formación profesional durante tres años y medio, entre 2016 y 2019. Las acciones investigativas se organizaron de forma infusiva en el marco de las materias curriculares trabajadas con el grupo durante ese periodo.

El proceso de investigación transcurrió en cinco etapas, con objetivos y tareas específicas, pero estrechamente relacionadas entre sí (ver figura 1).

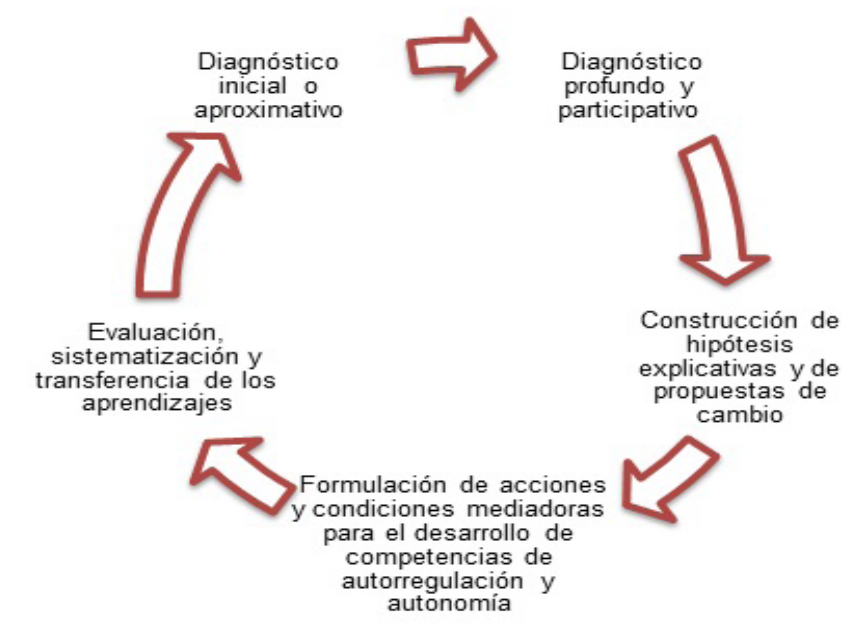

Figura 1. Etapas del proceso de IAP con estudiantes de la licenciatura en Intervención Educativa, UPN. 
Es importante destacar que el diseño de la IAP se articuló con el método genético propuesto por el enfoque histórico cultural de Vigotsky (1987), que permite la investigación de los fenómenos sociales y naturales a partir del análisis de su formación. De acuerdo con este autor, las cualidades y los contenidos de un fenómeno psicológico encarnan la trayectoria y dinámica de sus transformaciones y su naturaleza sociocultural. Por ello, su comprensión solo puede darse "mediante la consideración de la forma y el momento de su intervención en el curso del desarrollo" (Wertsch, 1995, p. 35).

Las técnicas utilizadas en el proceso de recuperación de datos fueron, en esencia, la observación participante, las autobiografías, los grupos focales, el diario de campo, cuestionarios, el inventario de autorregulación para el aprendizaje, de Lindner, Harris y Gordon (1996), las entrevistas apreciativas y generativas, y el diálogo permanente a nivel grupal.

Las técnicas utilizadas para el análisis de los resultados se corresponden en lo fundamental con aquellas sugeridas para la interpretación de datos cualitativos por su predominio en la investigación. Empleamos, así, procedimientos de organización, categorización y codificación de estos y fue medular el uso de la técnica de triangulación de datos para la validación de la información y las evidencias, así como para la confrontación crítica de los resultados. Ello impidió que se asumiera directamente su validez y se lograra mayor claridad en las categorías desarrolladas en el curso de la investigación (Glaser \& Strauss, 1967).

Con relación a la tareas de análisis de la información, al inicio del estudio llevamos a cabo un proceso de categorización descriptiva (Gibbs, 2012) apoyado en una codificación más específica que identificó contenidos y fragmentos relevantes que pudieran conceptualizarse desde categorías previas, así como otras categorías con el contenido emergente, que fueron necesarias para denominar la nueva información, sobre todo relacionada con los tipos de factores que habían incidido en las trayectorias formativas de sus procesos de autorregulación y autonomía o respecto de sus percepciones acerca de los problemas enfrentados para desarrollarlos.

En un momento posterior, identificamos y propusimos categorías más integradoras a partir de un proceso de categorización y "codificación más analítica y teórica" (Gibbs, 2012), en el cual fue necesario hacer inferencias profundas con base en los datos obtenidos. Para ello, efectuamos una interpretación y elaboración compleja en busca de relaciones, tendencias y generalizaciones, por ejemplo, acerca de condiciones mediadoras de la formación de la autorregulación y la autonomía encontradas en el análisis de la historia de su desarrollo en los aprendices y de las acciones y los procesos psicopedagógicos mediadores de esa formación.

La presentación de los resultados y su integración condujo a una narrativa cualitativa de la experiencia de IAP que se articuló a través de los principios organizativos de la sistematización. En correspondencia, esta última se llevó a cabo en cinco momentos esenciales de análisis: el punto de partida o situación inicial; la presentación de los cuestionamientos iniciales; la recuperación del trayecto vivido y sus resultados; la reflexión profunda y crítica; y los aspectos de llegada o saberes obtenidos, considerados estos últimos los conocimientos producidos y aprendizajes logrados (Jara, 1994).

La abstracción de tales saberes y la conceptualización final que condujo a la respuesta de la pregunta general de este estudio se alcanzaron con la teoría 
fundamentada, que, como "estrategia interpretativa" (Padilla, 2010, p. 157), permitió la generación de nuevas explicaciones teóricas y la construcción de conocimiento (Corbin, 2010); además, se articuló en este proceso de sistematización como base para una teorización más amplia y profunda (Jara, 2018).

\section{Participantes en el estudio}

Los participantes de la investigación fueron los estudiantes de la generación 20152019 de la licenciatura en Intervención Educativa de la UPN en la sede Ayala, Morelos; la muestra se conformó de 32 aprendices (10 hombres y 22 mujeres), con un rango de edad entre los 19 y 22 años. El grupo fue elegido por constituir la generación de más reciente ingreso a la licenciatura, lo que brindó la posibilidad de lograr una trayectoria investigativa longitudinal y fomentar un proceso de investigación acción verdaderamente participativo.

\section{MODELOS EXPLICATIVOS DE LA AUTORREGULACIÓN Y LA AUTONOMÍA EN EL CONTEXTO DE LA PSICOLOGÍA EDUCATIVA CONTEMPORÁNEA: LAS APORTACIONES DEL ENFOQUE HISTÓRICO CULTURAL}

Las propuestas teórico-metodológicas de la psicología de la educación en la actualidad promueven el aprendizaje autónomo y permanente en los diferentes niveles educativos (Woolfolk, 2010; Pozo, 2008). Como tendencia, se destaca la necesidad de fortalecer la implicación personal y el compromiso de la persona que aprende en su propio aprendizaje.

Desde un punto de vista psicoeducativo, la autonomía en el ámbito del aprendizaje remite a la capacidad de aprender a aprender; es comprendida como "[la] facultad de tomar decisiones que permitan regular el propio aprendizaje para aproximarlo a una determinada meta, en el seno de unas condiciones específicas que forman el contexto de aprendizaje" (Monereo, 2008, p. 12).

Monereo y sus colaboradores (2008) aseguran:

En síntesis, podríamos afirmar que lograr que nuestros alumnos sean más autónomos aprendiendo, es decir, que sean capaces de autorregular sus acciones para aprender, implica hacerlos más conscientes de las decisiones que toman, de los conocimientos que ponen en juego, de sus dificultades para aprender y del modo de superar esas dificultades (p. 12).

En específico, el estudio que este documento presenta retoma estos presupuestos para esclarecer y proponer las acciones mediadoras que, desde el plano educativo y pedagógico, podrían contribuir al desarrollo de los procesos de autonomía y autorregulación. Como señalan Pozo y Monereo (2002), "si tuviéramos que elegir un lema, un mantra que guiara las metas y propósitos de la escuela del siglo XXI, sin duda el más aceptado [...] entre educadores e investigadores [...] sería el que la educación tiene que estar dirigida a ayudar a los alumnos a aprender a aprender" (p. 11).

En esa dirección, es importante comprender no solo lo que significa la autonomía en el aprendizaje, sino los mecanismos que favorecen la autorregulación, dada la profunda imbricación entre ambos procesos. 
De acuerdo con Panadero y Alonso-Tapia (2014), podemos distinguir siete grandes teorías sobre autorregulación en el contexto de la psicología educativa: la teoría del conductismo operante, la fenomenológica, la del procesamiento de la información, la sociocognitiva, la volitiva, la vigotskiana y la constructivista. Según estos autores, las aportaciones comunes de todas ellas giran en torno a cinco aspectos fundamentales para entender la autorregulación: el origen de la motivación para autorregularse; la adquisición de la conciencia necesaria para hacerlo; la determinación de los procesos autorregulatorios básicos; el papel del entorno social y físico en la autorregulación del alumno; y las formas de adquisición de la capacidad para autorregularse.

Desde la perspectiva concreta de la teoría sociocognitiva, la autorregulación está estrechamente ligada a la elección del aprendiz respecto de sus recursos disponibles para aprender, siempre y cuando las condiciones externas le permitan asumir el control, por ejemplo, de las estrategias que emplea el docente. "El potencial de autorregulación varía dependiendo de las opciones de que dispongan los aprendices" (Schunk, 2012, p. 406).

Dicho en otras palabras, un aprendiz tiene oportunidad de autorregular su aprendizaje cuando los aspectos que conforman la tarea de aprendizaje no están del todo controlados por el profesor. "Las posibilidades de autorregulación varían de bajas a altas dependiendo de cuántas elecciones tengan los aprendices" (Pintrich \& Schunk, 2006, p. 171), es decir, de sus posibilidades de tomar decisiones de manera autónoma.

En esta investigación, en particular, tomamos como presupuestos básicos los principios del enfoque histórico-cultural, nacido con la obra de Vigotsky, a los que se articulan las principales aportaciones de los modelos antes mencionados. A partir de ese enfoque, asumimos la génesis social de los procesos psicológicos de tipo superior y otorgamos un papel relevante a la enseñanza en su formación. A través de la formulación de la ley genética general del desarrollo cultural, Vigotsky (1987) explica la transición hacia el desarrollo en el contexto de la zona de desarrollo próximo:

El contexto social y los instrumentos de mediación son los que, a través de los procesos de interiorización, poseen un carácter formativo sobre los procesos psíquicos superiores. Es el dominio progresivo e interiorizado de los instrumentos de mediación, de los sistemas de representación disponibles y en uso en el escenario social, el indicador por excelencia de los cambios y progresos del aprendiz (Rodríguez-Mena, 2008, p. 89).

Esta es la misma razón por la que Wertsch (1995) señala que el desarrollo se describe como la apropiación progresiva de nuevos instrumentos de mediación o como el dominio de formas más avanzadas de tales instrumentos: solo a través de estos mecanismos se logra la reorganización psicológica que da sentido de progreso al desarrollo psíquico.

De esa manera, la concepción vigotskiana fundamental del desarrollo conduce a la comprensión de la autorregulación como formación psicológica cualitativamente superior, como cualidad y meta del desarrollo psicológico humano. El hombre se apropia de los instrumentos culturales que le permiten transformar su mente y, en consecuencia, realizar funciones psíquicas intrapersonales que le garantizan la autorregulación de sus aprendizajes y su propio desarrollo, pues se convierten en mediadores psicológicos que le proporcionan posibilidades ilimitadas de autodesarrollo para, a su vez, continuar apropiándose de la cultura (García, 2012). 
Con base en las propuestas conceptuales referidas, en el estudio se entiende que

... la autorregulación del aprendizaje implica un modo de aprender independiente y activo regido por objetivos y metas propios; supone el dominio y aplicación planificada y adaptable de recursos y procesos, referidos a estrategias metacognitivas, estrategias cognitivas y procesos de dirección y control del esfuerzo, así como de componentes motivacionales, los que, en su conjunto, permiten resultados valiosos en los disímiles contextos en los que se inserta la "persona-que-aprende". Constituye un proceso complejo de carácter superior que se forma socialmente y se fortalece a lo largo del desarrollo de la personalidad de la persona-que-aprende (Rodríguez-Mena, García, Corral y Lago, 2004, p. 15).

Los procesos de autonomía y autorregulación del aprendiz se desarrollan a partir de la conformación de tres núcleos de competencias que se expresan de forma articulada: competencias para la estructuración, competencias para la contextualización y competencias para la gestión de las experiencias de aprendizaje (Rodríguez-Mena, García, Corral y Lago, 2004).

\section{EL ESTUDIO DE LA PERCEPCIÓN Y LAS OPINIONES DE LOS PARTICIPAN- TES RESPECTO A LAS NECESIDADES DE FORMACIÓN DE SUS PROCESOS DE AUTORREGULACIÓN Y AUTONOMÍA: PROBLEMÁTICAS QUE ENFRENTAN PARA SU DESARROLLO}

Durante las etapas de diagnóstico inicial y profundo de la investigación, promovimos la reflexión y el análisis vivencial para determinar las problemáticas que debían ser transformadas y reconocer las necesidades de los aprendices para ser autorregulados y autónomos. Los resultados presentados se obtuvieron de los cuestionarios, los grupos focales, las autobiografías, las sesiones de diálogo y la observación participante. Los comentarios y las opiniones de los jóvenes fueron codificados para respetar el acuerdo de confidencialidad.

La valoración de las problemáticas y necesidades referentes a la formación de los procesos de autonomía y autorregulación del aprendizaje se llevó a cabo a partir de dos ejes:

-Problemáticas y necesidades en torno a la comprensión de la autonomía y autorregulación y su conceptualización

-Problemáticas y necesidades relacionadas con los indicadores de autonomía y competencias de autorregulación: de estructuración, gestión y contextualización del aprendizaje

Problemáticas y necesidades en torno a la comprensión de la autonomía y autorregulación y su conceptualización

La determinación de estas problemáticas se realizó tomando en cuenta las categorías mostradas en la figura 2 . 


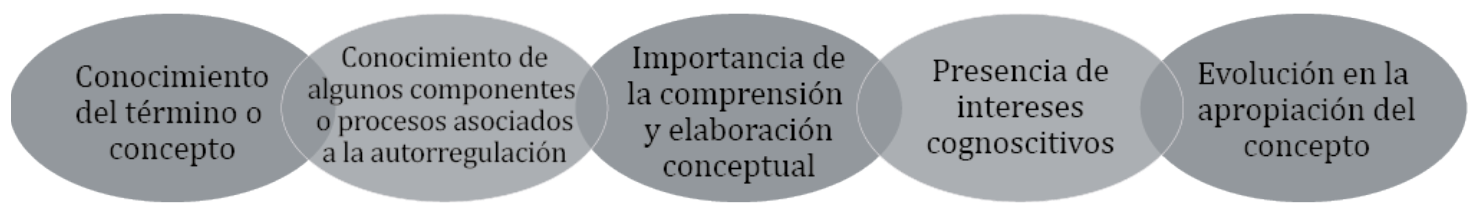

Figura 2. Categorías para el análisis de las problemáticas relacionadas con la comprensión de los procesos de autonomía y autorregulación y su conceptualización.

Conocimiento de los términos

Corroboramos el desconocimiento del término autorregulación y la expresión de preconceptos o ideas espontáneas sin sustento científico en la mayoría de los participantes, que indicaban la ausencia de una estructura cognitiva pertinente acerca del tema. De igual forma, se manifestaron pocas nociones en torno a la autonomía, ligadas, con frecuencia, a la idea de independencia. Expresiones como esta indican el desconocimiento de estos conceptos: "No, no sabía, de hecho, estoy leyendo mis respuestas y están raras porque yo no conocía, o bueno desconozco un poco sobre el tema de autorregulación" (S15).

Conocimiento de algunos componentes o procesos asociados a la autonomía y la autorregulación

Algunos aprendices mostraron que ya tenían ideas pertinentes sobre los procesos estudiados, las cuales constituían elementos esenciales para su comprensión, aunque apreciamos que eran parciales o incompletas. No obstante, la manifestación de esas ideas fue un momento cognitivo importante para la construcción grupal que permitió enriquecer la representación de los participantes acerca de los procesos analizados y los componentes implicados en ellos. Ejemplo de esto es la siguiente opinión: "Yo igual pienso que buscar las estrategias, es lo que ya había puesto, es una forma autónoma buscando nuestras propias estrategias de aprendizaje y pienso cada quien trata de plantearse los objetivos que quieren cumplir..." (S 27).

Importancia de la comprensión y elaboración conceptual (necesidad de conocimiento de los significados abordados)

Los datos registrados al inicio del estudio reflejaron una percepción limitada de los aprendices sobre la importancia de ser autónomos y autorregulados y de conocer dichos procesos. Sin embargo, al preguntar en uno de los grupos focales si sería preocupante para ellos no saber qué era la autorregulación, surgieron también posturas interesantes respecto a nuevos motivos cognoscitivos:

... porque no puedes ser algo de lo que no conoces, o sea no puedes ser autorregulado, si no sabes qué es autorregulado, entonces igual y si ya con lo que hemos visto que se necesita para poder tener la autorregulación, igual y si tenemos algunas cosas, pero no 
todas en sí, en su totalidad, entonces creo que es indispensable y primordial tener bien claro qué es, ser un estudiante autorregulado, un aprendiz autorregulado (S15).

\section{Presencia de intereses cognoscitivos}

Este aspecto fue relevante para identificar si los estudiantes manifestaban indicios de acciones de búsqueda de información para clarificar sus ideas, o ampliar y reestructurar los significados de autonomía, autorregulación y de aprendiz autorregulado. Los resultados indicaron que la búsqueda de información acerca de los procesos de autorregulación y autonomía no constituyó un motivo cognitivo predominante en muchos de los estudiantes, al menos hasta ese momento, a pesar de que se estaba abordando el tema durante la investigación acción y dialogando al respecto. Ello constituyó un punto de partida para la introducción de nuevas estrategias de reflexión y motivación, dirigidas a estimular nuevos intereses cognitivos.

\section{Problemáticas y necesidades relacionadas con los indicadores de las competencias de autorregulación}

En el diagnóstico profundo, los estudiantes pudieron descubrir problemáticas y ahondar en la percepción de necesidades en cuanto al desarrollo de los tres núcleos de competencias para la autonomía y la autorregulación: estructuración, gestión y contextualización.

Respecto a la competencia de estructuración, los resultados revelan la presencia de varios aspectos que tienen que ver con sus indicadores y que se vinculan a las categorías de la figura 3.
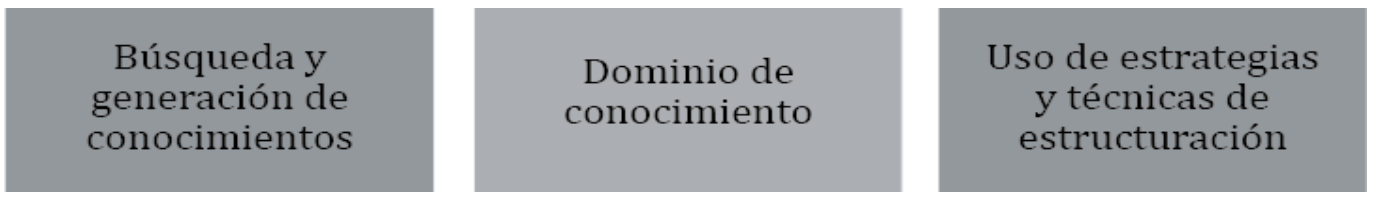

Figura 3. Categorías para el análisis de las problemáticas relacionadas con las competencias de estructuración.

\section{Búsqueda y generación del conocimiento}

Las principales problemáticas de esta categoría se asociaron a la posibilidad de hacer interpretaciones adecuadas de la información para estructurar los saberes acerca de la autorregulación y los conocimientos en general en las distintas materias:

Yo creo que cuando no entiendo algo en las clases y le pregunto a mis compañeras y vemos que no le entendimos, entonces más que nada, igual, se me complica entender las cosas ¿no? y entonces, si no me saben explicar; yo por mi parte debo investigar ¿no? y no quedarme con lo que dicen los profesores, sino investigar para saber bien y no quedarme así, y decir que no le entendí (S6).

Es importante destacar que los aprendices reconocen que tienen necesidad de trabajar procedimientos para la búsqueda y selección de información en diversas fuentes. Algunas cuestiones suponen el manejo de habilidades básicas de comprensión y, en 
particular, de comprensión lectora, así como de elección de información pertinente para la estructuración de saberes; otras necesidades están vinculadas al manejo de habilidades más complejas de pensamiento crítico y reflexivo para el análisis y la construcción de sus ideas.

\section{Dominio de conocimiento}

Perciben que no tener conocimiento al respecto limita sus decisiones y metas de aprendizaje para ser aprendices autorregulados, pero lo interesante es que el diálogo les permitió comprender que esta situación trasciende a cada experiencia de aprendizaje. Si no poseen conocimientos previos, se hace difícil la elaboración de otros nuevos, por lo que se debe buscar de forma intencionada el dominio del saber para seguir aprendiendo.

Fue interesante el descubrimiento de la necesidad de hacer análisis metacognitivo y reconocer qué han aprendido y cómo utilizar sus conocimientos en nuevas situaciones, por ejemplo, en prácticas profesionales o en la construcción de sus proyectos de desarrollo educativo para titularse. Revelaron que muchas veces no establecen relaciones con lo aprendido ni buscan sus apuntes o la información ya obtenida.

Uso de estrategias y técnicas de estructuración

Pudimos apreciar que los estudiantes poseen escasos saberes vinculados al dominio teórico y procedimental de estrategias de estructuración y organización del conocimiento, pues estas no son mencionadas con precisión y, por lo general, se alude solo a que se requieren métodos y técnicas que sean relevantes para cada aprendiz sin distinguir tipos o formas de utilización. No obstante, se destaca el reconocimiento del papel fundamental de las estrategias para construir el conocimiento y para la autorregulación del aprendizaje:

Y creo que también como dice, el interés, y depende de ello los métodos o técnicas que tú adquieras para regular tu aprendizaje, para buscar más ideas, más conceptos, que te queden más claros, depende mucho también de lo que tú hagas, los métodos o las técnicas que consideres que son importantes para ti, aunque, por ejemplo, para otras personas puede que no funcionen, pero para ti sí, entonces es buscar la manera de ampliar tu aprendizaje, buscar la mejor manera en que tú aprendas (S26).

En términos generales, tanto en los grupos focales como en momentos posteriores de trabajo, el grupo reconoció la necesidad de abordar, de forma explícita y amplia, las estrategias de aprendizaje para identificarlas y apropiarse de ellas. Mencionaron el hecho de no poseer experiencias previas de aprendizaje de tales procedimientos y de que, durante años, los han empleado de forma intuitiva, de acuerdo con las orientaciones superficiales de sus maestros o modelos inapropiados e ingenuos.

Respecto a las competencias para la gestión, se destacan las siguientes problemáticas conforme a las categorías de análisis empleadas, las que se presentan en la figura 4. 

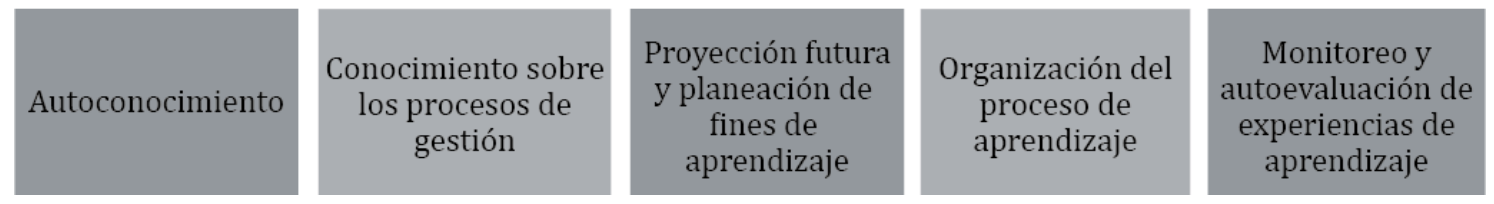

Figura 4. Categorías para el análisis de las problemáticas relacionadas con las competencias de gestión.

Autoconocimiento

Varios estudiantes hicieron comentarios directamente de esta categoría sobre la importancia de poner en marcha su autoconocimiento; también mostraron sus carencias al respecto e indicaron que muchas veces está ausente en el marco de sus experiencias de aprendizaje. "Ni tampoco sabemos sobre nosotros mismos para poder hacer una tarea bien, por ejemplo: el ambiente, con qué música te concentras. Entonces necesitamos conocernos nosotros mismos para tener un aprendizaje mejor" (S14).

El reconocimiento de fortalezas y habilidades para aprender constituye otra cuestión relevante en el proceso de autoconocimiento, pues este elemento es fundamental en la toma de decisiones sobre metas personales, las cuales deben ser acordes con sus posibilidades personales: "Pues, ya somos ahora sí, como se podría decir, ya somos estudiantes de universidad, ya no somos como antes en la primaria que nuestros papás nos tenían que decir: '¡Haz la tarea!, ¡estudia!', sino que ahora sí nosotros tenemos el propio interés en querer, ahora sí, aprender” (S29).

Es importante señalar que los estudiantes reconocen que poseen fortalezas que tienen que ver con sus motivaciones, disposición para aprender, interés, pero son menos capaces de percibir con claridad e identificar sus habilidades para guiar y lograr sus aprendizajes, en especial cognitivas, metacognitivas o procedimentales.

\section{Conocimiento sobre los procesos de gestión}

Entre las categorías que expresan este conocimiento, se encuentran la conceptualización y caracterización de las metas y el reconocimiento de valores y actitudes necesarios para lograr el aprendizaje. Respecto al primero, no aparecieron evidencias explícitas en el análisis; sin embargo, en el marco de las primeras materias trabajadas, los estudiantes mostraron dificultades para definir y caracterizar las metas de aprendizaje. Desde el punto de vista conceptual, manejaban indistintamente los conceptos de expectativas, metas, objetivos pedagógicos y propósitos.

Con relación al reconocimiento de valores y actitudes para el aprendizaje, destaca la aparición de esta categoría de manera emergente en la discusión grupal. Los estudiantes resaltaron valores y posturas éticas necesarios para la autorregulación y su desempeño autónomo, lo cual resulta un aspecto interesante en el que no se ha puesto suficiente interés en la investigación sobre autorregulación, y se ha dejado implícito en el terreno de los componentes motivacionales del proceso. Sin embargo, la existencia de valores sociomorales como motivos dominantes en la personalidad (Bozhovich, 1972; González, 1985) podría ser crucial para la de- 
terminación de la orientación de las metas propias y la implicación de los jóvenes en la búsqueda de calidad en sus aprendizajes. Al respecto, los jóvenes señalan la importancia de la responsabilidad y la honestidad consigo mismos y con los demás como componentes necesarios para autorregularse.

Proyección futura y planeación de fines de aprendizaje

La totalidad de los aprendices coincidieron en que en muy pocas ocasiones elaboran sus metas en las situaciones de aprendizaje y que una dificultad bastante grave es que no tienen conciencia de sus fines cuando cursan las materias o que, en realidad, no se preocupan por ello. Esta cuestión afecta la toma de decisiones oportunas en el proceso de aprendizaje respecto a los modos de conseguirlo. Refieren que toman como meta solo el hecho de terminar o pasar la materia o, en el mejor de los casos, asumen los objetivos que sus maestros les propongan. Ante la pregunta de si poseían metas de aprendizaje, la mayoría refirió que no las elaboran: "Yo la verdad, así como tal no, no tengo como esa claridad, o sea, sí me pongo la meta de querer terminar, por ejemplo, una materia, investigar y aprender, pero como tal no tengo como esa forma de decir, ¡ah! bueno qué trata de decir tal materia o cuál es el fin, o sea, no tengo mucho esa claridad al inicio" (S21).

En general, pudimos advertir que no se construyen metas de forma sistemática, es decir, se carece de fines generados de manera consciente que orienten con regularidad los procesos de aprendizaje; se hace ocasionalmente de modo espontáneo, en la medida que identifican intereses propios vinculados a las materias. Ello indica la falta de orientación y de visión anticipada de los logros esperados durante la formación académica.

Para la proyección futura y la planeación del proceso de aprendizaje, no solo es importante elaborar los fines personales y grupales, sino lograr la jerarquización de metas y su estructuración temporal. Según los datos obtenidos, por lo general, estas acciones de jerarquización se llevan a cabo como un proceso esporádico y ligado a las circunstancias de presión académica, cuando tienen que entregar trabajos o responder a situaciones problemáticas. No se anticipa o planean las metas con orden, por lo que puede inferirse que la organización de las tareas y del tiempo también se ve afectada. En el ejemplo siguiente se manifiesta esa preocupación: "Priorizar, porque a veces queremos hacer cosas al mismo tiempo que van encaminadas a cumplir metas distintas, pero pueden que algunas sean más importantes que otras que tengamos [...] Hay que tener claro qué meta se subordina a la otra y cuál puede atrasarse un poquito" (S16).

La situación encontrada tiene que ver con la posibilidad y el uso de estrategias para la autonomía y para tomar sus decisiones propias y fundamentadas, tanto en las actividades escolares específicas como en aspectos más complejos, como su futuro laboral. De ese modo, se manifiesta la necesidad de aprender a buscar información, valorar sus potencialidades y fortalezas, y definir metas claras con relación a su aprendizaje y su futuro profesional sobre la base del pensamiento crítico y la argumentación.

Organización del proceso de aprendizaje 
Un elemento fundamental para la organización del proceso de aprendizaje es la distribución y administración del tiempo. Este punto fue, quizá, uno de los más abordados por los aprendices y en el cual identificaron mayor número de problemáticas. Como tendencia, expresan deficiencias y problemáticas en el manejo del tiempo para planear sus tareas de aprendizaje y realizarlas. Reconocieron que se les dificulta decidir cuánto tiempo requieren para las distintas actividades, lo que provoca que haya desproporción en su distribución o no les alcance para cumplir con todas sus tareas. Como resultado de sus reflexiones, concluyeron que la organización y administración del tiempo es un aspecto que debían fortalecer, para lo cual necesitaban aprender técnicas y desarrollar habilidades autorreguladoras: "Pues algunas sí, pero con lo del tiempo no, soy muy confiado con el tiempo, luego digo 'sí esto lo hago' y no, cuando veo ya es bien tarde y no he hecho nada..." (S1).

\section{Monitoreo y autoevaluación de experiencias de aprendizaje}

Los participantes ofrecieron evidencias de estos indicadores de las competencias de gestión en especial relacionados con la necesidad de tomar en cuenta errores y fallas al aprender y desplegar acciones de mejora y autocorrección en la búsqueda de sus metas de aprendizaje. De igual manera, perciben la necesidad de ser flexibles y estar abiertos a observaciones y sugerencias que otros puedan ofrecer para reencauzar sus acciones y tareas, en particular cuando los docentes les hacen revisiones de sus tareas: "Si no entregamos los trabajos que nos hacen falta, no nos regulamos y no volvemos a revisar y verificar qué es lo que está mal o está bien, solo nos importa la calificación y ya" (S9).

Los jóvenes perciben que requieren fomentar la autorreflexión para percatarse de los factores que en la historia de su desempeño como aprendices han limitado su desarrollo como estudiantes autorregulados, de los problemas que afectan su formación y de la necesidad de ser conscientes de las nuevas metas que ahora deben lograr para serlo:

A mí también me gustó y ese es un punto importante, lo de la autorreflexión porque ahorita ya también pensándolo bien y desmenuzando como dice P [...], me di cuenta que esto de la autorregulación o sea es importante sí, pero como que es un proceso que tenemos que desarrollar desde pequeños y creo que no se nos dio la oportunidad y sé que no es muy bueno regresar al pasado ni nada de eso, pero te das cuenta de dónde empieza el problema de por qué no tienes la autorregulación bien desarrollada a este nivel, porque desde lo básico, desde la educación básica nunca se te dio esa oportunidad de tú tener la autonomía de aprender cosas (S22).

Respecto a las competencias de contextualización, se destacaron algunas problemáticas relacionadas con las categorías de la figura 5.
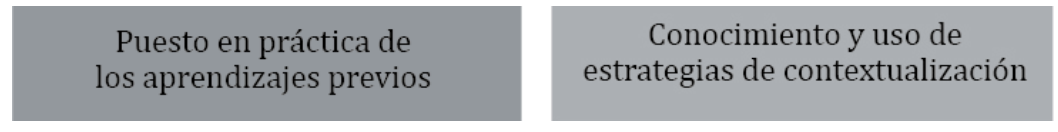

Figura 5. Categorías para el análisis de las problemáticas relacionadas con las competencias de contextualización. 
Puesta en práctica de los aprendizajes previos

Los aprendices mostraron dificultades para retomar sus conocimientos anteriores en situaciones nuevas de aprendizaje de manera intencional. Una hipótesis explicativa frente a estas dificultades podría vincularse a que "lo aprendido" no pueda considerarse un buen aprendizaje, dado que, para ser transferido este, debe ser el resultado de cambios duraderos (Pozo, 2008). Otra razón podría suponer la disposición misma de los aprendices a relacionar sus saberes con la nueva información (Ausubel, 2002) y, en consecuencia, a contar con habilidades de cognitivas y metacognitivas para activar con oportunidad sus conocimientos previos y poder transferirlos.

De cualquier manera, los jóvenes mostraron dificultades en estos aspectos y reconocen la necesidad de tomar conciencia de sus conocimientos y garantizar que estos sean bien elaborados para poder contar con ellos posteriormente.

Conocimiento y uso de estrategias de contextualización

Las estrategias de contextualización incluyen el empleo de analogías, el uso de ejemplos y contraejemplos, el empleo de comparaciones y la expresión de evidencias (Rodríguez-Mena, García, Corral y Lago, 2004); la manifestación de indicios en torno al manejo de estas estrategias no fue amplia, pero los estudiantes mostraron algunas expresiones, sobre todo referentes a la necesidad de utilizar ejemplos que clarifiquen lo que se aprende o hacer comparaciones que garanticen la distinción de similitudes y diferencias entre saberes y, en consecuencia, una mayor comprensión de ellos: "Quizá porque no sé reconocer el camino adecuado, porque hay veces que entiendo algo, pero [...] y trato de ejemplificarlo en otra cosa y con que no puedo, no puedo dar solución" (S15).

\section{Propuestas para CONTINUAR El PROCESO DE FORMACión Como APRENDICES AUTÓNOMOS Y AUTORREGULADOS: LAS ACCIONES GENERATIVAS DE LOS PARTICIPANTES}

Uno de los puntos tratados durante los grupos focales y la entrevista apreciativagenerativa, así como en otros espacios de diálogo grupal durante la IAP, fue la proyección de acciones, alternativas y posibles estrategias que el grupo podría llevar a cabo si se consideraba relevante desarrollar su autonomía y las competencias de autorregulación del aprendizaje. Estas propuestas fueron integradas en la etapa tres de construcción de hipótesis explicativas del estudio. A continuación, presentamos algunas de ellas, las cuales fueron categorizadas tras el análisis de las opiniones e ideas de los estudiantes.

\section{Propuestas de carácter procedimental y organizativo}

Los jóvenes destacan con fuerza la necesidad de contar con técnicas y estrategias tanto para el estudio como para la creación de metas, así como para organizar el tiempo y sus tareas escolares. Ejemplo de algunas de estas propuestas son las siguientes: 
-Lograr la organización del proceso de aprendizaje:

La organización del tiempo, hacer tus deberes, gestionando bien tus actividades personales, tener una postura propia que se requiere para ser un buen investigador. Con esfuerzo, priorizar cosas para llegar a un objetivo, ser organizado, responsable (S9).

-Promover la interacción y el diálogo permanentes:

Me he apoyado en mis compañeros de alto rendimiento, con los que he podido resolver mis dudas (S13).

\section{Propuestas de carácter cognitivo y metacognitivo}

Una de las propuestas de tipo cognitivo evidente fue la búsqueda de la conceptualización y profundización en la comprensión de los términos autorregulación del aprendizaje, aprendiz autorregulado y autonomía. Los estudiantes reconocieron la necesidad de abordar estos conceptos de manera explícita para comprenderlos y establecer las relaciones necesarias entre los significados que se estaban manejando. Destacaron, además, la conveniencia de buscar conocimientos para ser autónomos y tomar decisiones sobre todo relacionadas con su futuro profesional y con los contenidos que deben aprender. Algunos indicadores se manifiestan a continuación:

-Desarrollar el autoconocimiento y la metacognición para favorecer la autonomía:

Dándome cuenta de las habilidades que me falta desarrollar, motivándome para poder desarrollarlas y empleando estrategias más funcionales. De igual forma, conocerme mejor es un punto fundamental que considero que me ayudó para estructurar mejor lo que debo hacer para lograr lo que quiero (S16).

-Propiciar la autorreflexión y la autonomía:

Elaborar un proyecto de vida, tener métodos de autoevaluación y darse cuenta de qué está fallando y cambiar donde se está mal, es decir, toma de decisiones, autonomía. Reforzar conocimientos (S1).

-Favorecer el autocontrol:

La primera acción que llevé a cabo fue la autogestión para ver mis tiempos y espacios, gestionándolos y teniendo control de ello, trabajando de manera independiente para poder ser autónoma y planteándome de manera positiva las estrategias de aprendizaje (S21).

-Poner en marcha acciones de autoevaluación

Realizar una "evaluación” por preguntas: ¿qué metas tengo? ¿Cómo las realizaré? ¿Logré lo planeado? ¿Qué espero de este aprendizaje? (S11).

\section{Propuestas de carácter motivacional}

- Partir de necesidades propias y generar metas personales:

Bueno yo iba a decir, primero crear nuestras metas, pensarlas, crearlas y ponerlas en práctica, bueno hacer una planeación para poder lograrlas (S2).

-Establecer metas sobre la base de una motivación intrínseca: 
Ser consciente de lo que quiero, necesito y me hace falta. Empezando por definir mis metas, en ellas plantearme lo que quiero lograr, teniendo en cuenta actividades que quiero realizar para alcanzarlas y sobre todo ser comprometida con el hacer diario (S14).

Propuestas de carácter ético

-Ser consecuentes con los valores necesarios para la autonomía y la autorregulación:

Bueno pues, como decían también, el ser honesto ¿no? Planteando nuestras metas. Porque hay veces que nos planteamos algunas cosas que lo vamos a hacer y como no las realizamos, lo dejamos todo como al final (S10).

La reflexión por parte de los participantes para descubrir y abrir caminos de desarrollo fue esencial para identificar las acciones psicopedagógicas necesarias para mediar y favorecer la formación de aprendices autónomos y autorregulados en las condiciones de su práctica formativa.

\section{EL SISTEMA DE MEDIADORES GENERADO PARA EL DESARROLLO DE LOS PROCESOS DE AUTONOMÍA Y AUTORREGULACIÓN DEL APRENDIZAJE EN EL MARCO DE LA IAP}

La identificación y creación de un sistema de mediadores que posibilitaran la formación de los procesos de autonomía y autorregulación del aprendizaje constituye uno de los logros fundamentales de esta investigación, resultado de la cuarta etapa del proceso investigativo (formulación de acciones y condiciones mediadoras para el desarrollo de competencias de autorregulación y autonomía). Entre estas acciones psicopedagógicas mediadoras, destacan:

-Empleo de acciones para la problematización, que permitan el desarrollo de habilidades de pensamiento para la identificación y verificación de problemáticas concernientes al desarrollo de la autonomía y la autorregulación, y posibiliten la valoración por parte de los participantes de sus causas y consecuencias en el aprendizaje.

-Utilización de recursos psicosociales para la sensibilización, que contribuyan al diseño de instrumentos de análisis de vivencias, percepción de hechos $\mathrm{y}$ contradicciones de sus historias personales y trayectorias escolares (elaboración y análisis de autobiografías), identificación de estados emocionales y afectivos generados por los procesos y vivencias construidas ante la imposibilidad de autonomía y autorregulación en el contexto escolar.

-Estimulación del análisis metacognitivo, identificación del origen de sus necesidades cognitivas y de sus saberes, así como el reconocimiento de las maneras en que fueron elaborados. Análisis de sus experiencias de autonomía y autorregulación, y de los procesos, habilidades y conocimientos que las hicieron posibles. Valoración de sus necesidades formativas para convertirse en aprendices autónomos y autorregulados. 
-Empleo de acciones de diálogo reflexivo, constructivo y argumentativo, que fomenten procesos de apertura, conflicto cognitivo y sociocognitivo para el análisis crítico de las oportunidades que ofrece la autonomía y la autorregulación en las condiciones formativas que brindan las instituciones educativas.

-Estimulación a la reflexión y autorreflexión como vía para pensar de manera crítica acerca de su formación profesional, sus actitudes como aprendices, sus experiencias de aprendizaje, sus perspectivas futuras y las alternativas para tomar decisiones adecuadas en el contexto educativo, así como para estructurar o reconstruir su proyecto de vida (como referente más amplio en la orientación de sus procesos de autonomía y autorregulación).

-Modelación y aplicación de estrategias de indagación sistemática, generación de interrogantes en el contexto de diálogo grupal, replanteamiento de preguntas para lograr la profundización en el análisis y la expansión del debate, así como para guiar el aprendizaje en torno a los procesos de autorregulación y autonomía.

-Diseño y aplicación de estrategias motivacionales para la elaboración de metas de aprendizaje. Propiciar la comprensión conceptual de las metas y la diferenciación de otros conceptos como expectativas, objetivos pedagógicos y propósitos. Empleo de técnicas para elaborar metas individuales y grupales activa y sistemáticamente en las materias. Favorecer el aprendizaje de estrategias de gestión para la construcción y planeación de metas y para alcanzar la interiorización de las acciones desarrolladas en el marco interactivo que sean convertidas en instrumentos propios de autogestión.

- Modelación de estrategias de aprendizaje con distintas funciones. Trabajar la explicación y construcción de sus pasos para favorecer el conocimiento procedimental a partir del principio de la necesidad de dominio para lograr la autorregulación y la autonomía. Trabajar en el plano teórico con materiales de apoyo (búsqueda del conocimiento de la estrategia en sí: origen, autores, características, componentes) y el plano práctico (aplicación metodológica para crear productos concretos en las clases y comprender los modos de utilización de acuerdo con las exigencias del aprendizaje, recursos necesarios de elección o ajuste, entre otras condiciones operativas).

-Construcción guiada de estrategias de autoevaluación. Punto fundamental del proceso mediador para aprender a autovalorarse, implica el análisis de su finalidad, así como de sus diferentes alcances y momentos. El referente esencial es la consecución de sus metas de aprendizaje y una acción mediadora clave supone la creación conjunta de los criterios y posibles indicadores para determinar sus logros.

- Creación de ambientes cooperativos, abiertos, flexibles y generadores de espacios para el error y la duda, como recursos para el aprendizaje. Negociación colectiva de la explicación de los errores y el rastreo de sus orígenes para ayudar al cambio y la corrección propia y grupal. Propiciar la construcción de una comunidad de aprendizaje como mediador social y el trabajo con estrategias cooperativas para la interacción constructiva.

Podríamos afirmar que la labor educativa sistemática como resultado de la IAP y el manejo explícito de mediadores psicopedagógicos, primero en el plano externo, 
posibilitó la interiorización de tales recursos por parte de los aprendices, que se convirtieron en instrumentos psicológicos internos que les ayudaron a regular sus propias acciones de aprendizaje.

\section{REFLEXIONES FINALES}

El proceso de IAP mostró que la formación de competencias para la autorregulación y la autonomía puede lograrse en el marco de la historia del desarrollo de estos procesos, como resultado de la puesta en marcha de los mediadores psicopedagógicos oportunos congruentes con su zona de desarrollo próximo y tomando en cuenta las condiciones psicológicas, históricas y socioculturales propias de los estudiantes.

En consecuencia, la transformación y el desarrollo de las competencias para la autorregulación y la autonomía requieren el autodescubrimiento por parte de los aprendices de sus propias necesidades formativas y la elaboración consciente de sus metas de desarrollo con relación a estos procesos. Los resultados avalaron el alto grado de sensibilización e implicación que pueden alcanzar los aprendices cuando son partícipes activos de las acciones de problematización referentes a su historia y papel como estudiantes y cuando pueden tomar decisiones con autonomía sobre sus propias alternativas de desarrollo, a diferencia de cuando son sujetos del diagnóstico o de investigaciones ajenas a su participación, lo cual ha sido una condición predominante en la investigación dentro de este campo (Rosário et al., 2013; Torrano, Fuentes y Soria, 2017).

A lo largo de la IAP, los participantes fueron capaces de identificar problemáticas y necesidades propias, así como analizar las causas de sus dificultades para ser aprendices autorregulados. Reconocieron sus condiciones potenciales para lograrlo y generaron de manera creativa las alternativas de cambio relacionadas con las competencias de estructuración, gestión y contextualización de sus experiencias de aprendizaje, traducidas en propuestas para su desarrollo cognitivo y metacognitivo, motivacional e incluso ético.

En esa misma dirección, los aprendices expusieron la necesidad de reestructurar su proyecto de vida en cuyo marco podrían replantear sus metas de aprendizaje y desarrollo profesional, así como las acciones para conseguirlas de manera autorregulada. Para ello, destacaron su disposición a incrementar el autoconocimiento, el reconocimiento de cualidades propias y de preferencias cognitivas que les posibilitaran gestionar mejor sus procesos de aprendizaje; también, apreciaron la relación del autoconocimiento con la autonomía, al manifestar actitudes favorables para alcanzarla como vía para la toma de decisiones respecto a su futuro profesional.

Los jóvenes obtuvieron una comprensión más profunda de sus propios procesos de autonomía y autorregulación, y una mayor elaboración conceptual de estos, lo que les permitió relacionar sus significados con su aprendizaje y formación profesional y otorgar sentidos diferentes a sus experiencias como aprendices. Con ello, se logró el análisis metacognitivo de sus maneras de actuar y sus necesidades, la reconstrucción de sus metas, y la búsqueda de concientización y control acerca de los instrumentos de autorregulación para que pudieran emplearlos de manera autónoma en nuevos aprendizajes y en situaciones de autotransformación y transformación de su realidad educativa. 
La apropiación de estrategias y conocimientos acerca de los procesos de autorregulación se vio reflejada en las actividades académicas, en las cuales mostraron mayor autonomía al tomar decisiones sobre los modos de participación y realización de estas, así como en la transferencia de lo aprendido a nuevas situaciones de desempeño escolar, tal como se pudo apreciar durante la construcción y aplicación de sus proyectos de intervención y en sus procesos de titulación.

Para alcanzar el desarrollo de las competencias de autorregulación y autonomía, la investigación demostró que se requiere el diseño de situaciones de aprendizaje que permitan la formación de aprendices autorregulados y que el docente, como mediador social, debe organizar tales situaciones y orientar las acciones mediadoras que se necesiten de manera conjunta con los estudiantes. No se trata solo de que la situación de aprendizaje brinde opciones de elección al aprendiz (Pintrich \& Schunk, 2006; Schunk, 2012), sino que en ella se generen los procesos y condiciones que favorezcan la conformación de las competencias adecuadas para mediar la formación de aprendices autónomos y autorregulados. De ese modo, la identificación del conjunto de acciones mediadoras expuestas en los resultados se torna una de las aportaciones esenciales del estudio como recurso metodológico sustancial para lograr la transformación y el desarrollo de los aprendices.

La articulación de las tareas de docencia e investigación hicieron posible tanto la identificación, diseño y puesta en marcha de las acciones psicopedagógicas como su análisis reflexivo y validación con la participación del grupo de estudiantes, lo que exigió una permanente apertura mental y una postura constructiva y creativa para mostrar la voz de los aprendices y guiar el proceso investigativo sin entorpecer el desarrollo de sus propias expresiones de autonomía y autorregulación.

\section{REFERENCIAS BIBLIOGRÁFICAS}

Aguilar, V. (2020). Autorregulación docente. Modelos para el fortalecimiento e investigación de la docencia. México: Octaedro.

Ausubel, D. P. (2002). Adquisición y retención del conocimiento. Una perspectiva cognitiva. Barcelona: Paidós.

Bisquerra, R. (coord.) (2014). Metodología de la investigación educativa (4a. ed.). Madrid: Editorial La Muralla.

Bozhovich, L. I. (1972). Estudios de las motivaciones de la conducta de los niños y adolescentes. La Habana: Editorial Pueblo y Educación.

Corbin, J. (2010). La investigación en la teoría fundamentada como medio para generar conocimiento profesional. En S. Bénard (coord.). La teoría fundamentada: una metodología cualitativa. México: Universidad Autónoma de Aguascalientes.

García, I. (2012). Estrategias para la autorregulación del aprendizaje en estudiantes de educación superior. Tesis de doctorado. Centro de Investigación y Docencia del Estado de Morelos, Cuernavaca, Morelos.

Gibbs, G. (2012). El análisis de datos cualitativos en investigación cualitativa. Madrid: Ediciones Morata.

Glaser, B. G. \& Strauss, A. L. (1967). The discovery of grounded theory: Strategies for qualitative research. Chicago: Aldine. 
González, F. (1985). Psicología de la personalidad. La Habana: Editorial Pueblo y Educación.

Guzmán, M. G., Peña, M. y Ramírez, M. L. (2015). Acompañamiento de la formación práctica del futuro docente en la escuela normal. En Memorias electrónicas del XIII Congreso Nacional de Investigación Educativa (vol. 2, núm. 1, pp. 2015-2016). Recuperado de http://www.comie.org.mx/congreso/memoriaelectronica/v13/doc/2204.pdf

Jara, 0. (2018). La sistematización de experiencias: práctica y teoría para otros mundos posibles. Bogotá: Centro Internacional de Educación y Desarrollo Humano -Cinde.

Jara, 0. (1994). Para sistematizar experiencias: una propuesta teórica y práctica. San José, CR: Centro de Estudios y Publicaciones, Alforja.

Lindner, R. W., Harris, B. \& Gordon, W. (1996). The design and development of the Self-Regulated Learning Inventory: A status report. Presentado en la American Educational Research Association, Annual Convention, NY, Session 27/28, poster C7.

Monereo, C. (coord.) (2008). Ser estratégico y autónomo aprendiendo. Unidades didácticas de enseñanza estratégica para la ESO. Barcelona: Graó.

Padilla, M. R. (2010). La teoría fundamentada como estrategia interpretativa. La construcción de la categoría de "el prejuicio" en dos estudios de caso. En S. Bénard (coord.). La teoría fundamentada: una metodología cualitativa (pp. 157-177). México: Universidad Autónoma de Aguascalientes.

Panadero, E. y Alonso-Tapia, J. (2014). Teorías de la autorregulación educativa: una comparación y reflexión teórica. Revista Psicología Educativa, vol. 20, núm. 1, junio, pp. 11-22. http://dx.doi.org/10.1016/j.pse.2014.05.002

Pintrich, P.R. \& Schunk, D. (2006). Motivación en contextos educativos. Teoría, investigación y aplicaciones. Madrid: Pearson Educación.

Pozo, J. I. (2008). Aprendices y maestros. La psicología cognitiva del aprendizaje. Madrid: Alianza Editorial.

Pozo, J. I. y Monereo, C. (2002). El aprendizaje estratégico. Enseñar a aprender desde el currículo. Madrid: Aula XXI-Santillana.

Rodríguez-Mena, M. (2008). La naturaleza social de los aprendizajes. En G. Fariñas y L. Coelho (orgs.). Cadernos ECOS: Educação, Cultura e Desenvolvimento Humano (vol. II). São Paulo: Terceira Margem Editora Didática Ltda.

Rodríguez-Mena, M., García, I., Corral, R. y Lago, C. (2004). Aprender en la empresa. Fundamentos sociopsicopedagógicos del Programa de Formación de Aprendices Autorregulados en Comunidades de Aprendizaje. La Habana: Prensa Latina.

Rosário, P., Pereira, A., Högemann, J., Nunes, A. R., Figueiredo, M., Núñez, J.C., Fuentes, S. y Gaeta, M. L. (2013). Autorregulación del aprendizaje: una revisión sistemática en revistas de la base Scielo. Universitas Psychologica, vol. 13, núm. 2, pp. 781-797. doi:10.11144/Javeriana.UPSY13-2.aars

Schunk, D. H. (2012). Teorías del aprendizaje. Una perspectiva educativa. México: Pearson.

Torrano, F., Fuentes, J. L. y Soria, M. (2017). Aprendizaje autorregulado: estado de la cuestión y retos psicopedagógicos. Perfiles Educativos, vol. XXXIX, núm. 156, pp. 160-173, Recuperado de https://www.iisue.unam.mx/perfiles/numeros/2017/156 
Vargas, R. (2015). Acompañamiento formativo: una propuesta de formación para docentes, directivos, asesores de educación básica y superior en investigación educativa. En Memorias electrónicas del XIII Congreso Nacional de Investigación Educativa (vol. 2, núm. 1, pp. 2015-2016).

Vigotsky, L. S. (1987). Historia del desarrollo de las funciones psíquicas superiores. La Habana: Editorial Científico Técnica.

Wertsch, J. W. (1995). Vygotski y la formación social de la mente. Barcelona: Paidós.

Woolfolk, A. (2010). Psicología educativa. México: Pearson Educación. 